\title{
Neonatal Bladder Irritation Is Associated With Vanilloid Receptor TRPV1 Expression in Adult Rats
}

\author{
Jee Soo Park ${ }^{1}$, Hae Do Jung ${ }^{2}$, Young Sam Cho ${ }^{3}$, Mei Hua Jinn ${ }^{1}$, Chang Hee Hong ${ }^{1}$ \\ ${ }^{1}$ Department of Urology, Yonsei University College of Medicine, Seoul, Korea \\ ${ }^{2}$ Department of Urology, Yongin Severance Hospital, Yonsei University Health System, Yongin, Korea \\ ${ }^{3}$ Department of Urology, Kangbuk Samsung Hospital, Sungkyunkwan University School of Medicine, Seoul, Korea
}

Purpose: To evaluate whether mild chemical irritation of the bladder in neonatal rats is associated with persistent vanilloid receptor transient receptor potential vanilloid subfamily 1 (TRPV1) activity in adult rats.

Methods: Female Sprague-Dawley rats were used. Ten-day-old rat pups underwent bladder sensitization via intravesical infusion of $0.2 \%$ acetic acid in saline with or without prior bladder desensitization with capsaicin. After 8 weeks, 3 groups of rats (control [group 1], bladder sensitization [group 2], and bladder desensitization [group 3]) underwent cystometry. Inflammation of bladder tissue and the expression of TRPV1 in bladder tissue and dorsal root ganglia (DRG) were also evaluated.

Results: The bladder sensitization group showed more frequent voiding contractions. TRPV1 expression in adult bladder tissue was elevated in group 2. TRPV1 mRNA levels in the bladder and DRG were significantly higher in group 2 than in group 1. Moreover, group 2 had significantly more DRG neurons (identified by uptake of the retrograde label Fast Blue) that exhibited TRPV1 immunoreactivity.

Conclusions: We found a significant association between neonatal bladder sensitization and persistent TRPV1 activity in adult rats. This is the first study to focus on the underlying pathogenesis of bladder overactivity from childhood to adulthood. Our findings could lead to the development of new strategies for the treatment and prevention of adult urinary symptoms arising from childhood urinary tract dysfunction.

Keywords: Urinary bladder; Urinary tract infections; Interstitial cystitis; Capsaicin; TRPV cation channels

- Grant/Fund Support: This study was supported by a faculty research grant from the Yonsei University College of Medicine (6-2010-0063).

- Research Ethics: All procedures involving rats were approved by the Institutional Animal Care and Use Committee of the Yonsei University College of Medicine.

- Conflict of Interest: No potential conflict of interest relevant to this article was reported.

\section{- HIGHLIGHTS}

- Adult rats that experienced neonatal bladder sensitization showed more frequent voiding contractions.

- Adult rats that experienced neonatal bladder sensitization showed higher bladder and dorsal root ganglia TRPV1 mRNA levels and immunofluorescence.

- Neonatal bladder sensitization was significantly associated with increased TRPV1 expression.

Corresponding author: Chang Hee Hong (iD https://orcid.org/0000-0002-0946-7702 Department of Urology, Yonsei University College of Medicine, 50-1 Yonsei-ro, Seodaemun-gu, Seoul 03722, Korea

E-mail: chhong52@yuhs.ac / Tel: +82-2-2019-3473 / Fax: +82-2-3462-8887

Submitted: September 6, 2018 / Accepted after revision: September 13, 2018 (i) (7) This is an Open Access article distributed under the terms of the Creative Commons Attribution Non-Commercial License (http://creativecommons.org/licenses/by-nc/4.0/) which permits unrestricted non-commercial use, distribution, and reproduction in any medium, provided the original work is properly cited. 


\section{INTRODUCTION}

Several studies have found associations between childhood urinary symptoms and lower urinary tract symptoms in adult women [1]. For instance, a population-based cohort study of 2,109 participants reported significant associations between frequency, incontinence, and urinary tract infection during childhood and overactive bladder symptoms in adulthood [1]. The prevalence of interstitial cystitis or painful bladder syndrome, which is characterized by frequency and urgency, is 10 to 12 times higher in adults who experienced childhood urinary symptoms [2]. Several pathophysiological mechanisms of overactive bladder symptoms in adults have been suggested, including increased neuronal susceptibility, imbalance of bladder receptors, and altered smooth muscle cell junctions [1]. A genetic component has also been suggested $[3,4]$.

Several capsaicin-sensitive primary afferent neurons innervate the lower urinary tract. In particular, capsaicin-sensitive $\mathrm{C}$ fibers play an important role in voiding function. Capsaicinsensitive $\mathrm{C}$ fibers are both afferent fibers with sensory function and efferent fibers that release peptides such as substance $\mathrm{P}$ or calcitonin gene-related peptide. One capsaicin-gated ion channel protein is transient receptor potential vanilloid subfamily 1 (TRPV1). The use of TRPV1 antibody has revealed the anatomical distribution of unmyelinated $\mathrm{C}$ fibers innervating the bladder upon stimulation by bladder distention or chemical irritants, showing that TRPV1 is expressed in nerve fibers within the bladder muscularis, submucosa, and mucosa and appears in a functional form in transitional epithelial cells (i.e., urothelial cells) lining the bladder lumen $[5,6]$. TRPV1 is also involved in pathological bladder conditions. For example, Brady et al. [7] found that patients with neurogenic bladder overactivity exhibited increased expression of TRPV1. Furthermore, clinical studies have shown a significant association between increased TRPV1 expression and various pathological conditions of the bladder, such as neurogenic overactive bladder [8], nonneurogenic overactive bladder [9], interstitial cystitis or painful bladder syndrome [9], and hemorrhagic cystitis [10].

In this study, we investigated whether lower urinary tract dysfunction in childhood causes bladder overactivity in adults by injecting a mild chemical irritant into the bladder of neonatal rats and evaluating long-lasting changes in the bladder in adult rats. We also investigated whether TRPV1 is involved in the induction and maintenance of bladder sensitization. We showed that mild stimulation that does not cause significant inflamma- tion or injury can induce bladder sensitization and that TRPV1 plays a role in inducing and maintaining bladder sensitization.

\section{MATERIALS AND METHODS}

\section{Animals}

Rats were obtained as litters of 4-day-old pups. Thirty-six female Sprague-Dawley rats were divided into 3 equal groups: normal control rats injected with saline (group 1), rats with chemically-induced cystitis (group 2), and capsaicin-pretreated rats with chemically-induced cystitis (group 3). At the age of 8 weeks, cystometry was performed in 21 rats (7 rats per group). After cystometry, bladder tissues and dorsal root ganglia (DRG) were obtained in order to study inflammation in bladder tissue and the expression of TRPV1 in bladder tissue and DRG. Fifteen rats (5 rats per group) were injected with a retrograde tracer at 7 weeks to label bladder afferent neurons in the lumbosacral DRG. All procedures involving rats were approved by the Institutional Animal Care and Use Committee of the Yonsei University College of Medicine.

\section{Bladder Sensitization and Desensitization}

In the normal control group, postnatal day 10 (P10) rat pups were anesthetized with isoflurane and injected with $0.1 \mathrm{~mL}$ of saline. In the chemically-induced cystitis group, P10 rat pups were anesthetized with isoflurane, and $0.1 \mathrm{~mL}$ of $0.2 \%$ acetic acid solution in saline was injected into the bladder for bladder sensitization. In the capsaicin-pretreated chemically-induced cystitis group, $50 \mathrm{mg} / \mathrm{kg}$ capsaicin in 10\% ethanol, 10\% Tween 20 , and $80 \%$ saline was administered subcutaneously on P0 and re-administered after 24 hours (P1). Then, $0.1 \mathrm{~mL}$ of $0.2 \%$ acetic acid solution was injected into the bladder on P10 after isoflurane anesthesia.

\section{Cystometry}

After the onset of general anesthesia, the urinary bladder was exposed using a midline abdominal incision. A polyethylene catheter (PE-50) was inserted through a small incision in the bladder dome, followed by purse-string closure of the bladder. A PE-50 was connected with a t-stopcock to a micro-injection pump (Kd S-202, Stoelting Co., Wood Dale, IL, USA) and a pressure transducer with a PowerLab system (ADInstruments, Sydney, Australia). Variations in intraluminal pressure were recorded in response to continuous infusion $(0.12 \mathrm{~mL} / \mathrm{min})$ of saline solution to elicit rats' responses and to record bladder capacity, voided 
volume, residual volume, voiding efficiency, micturition pressure, and duration and frequency of premicturition contraction.

\section{Tissue Preparation and Harvest}

After the cystometry measurements were made, the urinary bladder was exposed through a median laparotomy, and bladder tissue was obtained and placed in $4 \%$ paraformaldehyde in phosphate-buffered saline (PBS). Cryoprotection was achieved by placing specimens in $20 \%$ sucrose/PBS. Using a microtome, $16-\mu \mathrm{m}$-thick cryosections were made and mounted on glass slides. Slides were stained with hematoxylin and eosin, cover slips were affixed, and they were inspected using light microscopy.

\section{Evaluation of Bladder Specimens for Inflammation}

Tissue samples for uses other than microscopic inspection were obtained after cystometry in order to quantify the proinflammatory cytokines interleukin (IL)- 6 and interferon (IFN) $-\gamma$. The bladder samples of 8 -week-old rats were obtained, weighed, homogenized, minced on an ice-cold plate, and resuspended in a centrifugation tube containing $10 \mathrm{mmol} / \mathrm{L}$ PBS. The tubes were placed in a shaker submerged in a $37^{\circ} \mathrm{C}$ water bath for 20 minutes and then centrifuged at $9,000 \times g$ for 30 seconds at $4^{\circ} \mathrm{C}$. The supernatants were frozen at $-80^{\circ} \mathrm{C}$ until assayed. IL- 6 and IFN- $\gamma$ levels were quantified using a DuoSet ELISA Kit (R\&D Systems, Inc., Minneapolis, MN, USA) to measure concentrations of natural and recombinant rat enzymes according to the manufacturer's instructions.

\section{Immunofluorescence Staining}

Tissue sections were incubated with a primary antibody against TRPV1 (1:200; PC420, Invitrogen, Carlsbad, CA, USA) at room temperature overnight. Sections were then washed with PBS (three 10-minute washes) and incubated with an appropriate secondary antibody (1:400; Alexa Fluor 546 goat anti-rabbit IgG, Molecular Probes, Eugene, OR, USA) for 2-4 hours. Labeled sections were observed and photographed with a Zeiss Axiophot fluorescence microscope equipped with epi-illumination and an appropriate filter set for fluorescein (FITC), Texas Red, and Fast Blue (FB) as well as a confocal microscope (Zeiss LSM 710, Oberkochen, Germany). Sections were collected from different representative regions of the DRG (i.e., the upper, middle, and lower thirds). To determine the percentages of particular neuronal populations, at least 300 FB-labeled neurons were examined. All traced cells found in particular sections were counted. To avoid double-counting of the same neu- rons, neurons were counted in every fifth section. Neuron counts were pooled for each rat. The quantity of TRPV1-labeled neurons was calculated as the percentage of TRPV1-labeled neurons relative to all FB-positive neurons counted.

\section{Quantitative Reverse-Transcription Polymerase Chain Reaction}

Total RNA was extracted from the same frozen DRG tissue that was used for immunofluorescence staining using RNeasy Protect Mini Kits (Qiagen, Hilden, Germany). Real-time polymerase chain reaction analysis was performed with an Applied Biosystems 5700 system using the TaqMan Master Mix (Applied Biosystems. Foster City, CA, USA). The following forward and reverse primer and probe sequences were used: TRPV1: GCAA GAAGCGCCTGACTGA, TGAGCATGGCTTTTAGCAGACA, TTTCCTGTCTCTGGGTCTTTGAACTCGCT; b-III-tubulin: GGGCCTTTGGACACCTATTCA, GCCCTTTGGCCCAGTTGT, CCTGACAACTTTATCTTCGGTCAGAGTGGTG. Relative changes in TRPV1 mRNA levels were calculated by the $\mathrm{Ct}$ method using $\beta$-III-tubulin as a normalizer.

\section{Retrograde Tracing of Bladder Afferent Cells}

At 7 weeks, the retrograde tracer FB (EMS-Chemie GmbH, Gross Umstadt, Germany) was used to label bladder afferent neurons in the lumbosacral DRG. After onset of general anesthesia, a total volume of $40 \mu \mathrm{L}$ of $4 \% \mathrm{FB}$ suspension was injected into each rat (8 injections of $5 \mu \mathrm{L}$ in each aspect) with a Hamilton syringe equipped with a $26-\mathrm{G}$ needle, taking particular care to avoid injection into the bladder lumen. Following each injection, to avoid leakage, the needle was left in place for 1 minute. The wall of the injected bladder was then rinsed with saline and gently wiped with gauze. After the evaluation of cystometry, the spinal cord was removed via dorsal laminectomy, the L6-S1 segments were retrieved, and the DRG, including the L6 and S1 DRG, were removed and postfixed by immersion in $4 \%$ paraformaldehyde in PBS for 1 hour and cryoprotected overnight in $20 \%$ sucrose/PBS. Transverse serial sections (16- $\mu \mathrm{m}$-thick) of each ganglion were cut using a cryostat. Slides were dried overnight at room temperature and subsequently stored at $4^{\circ} \mathrm{C}$ protected from light.

\section{Chemicals}

All chemicals were purchased from Sigma-Aldrich (St. Louis, MO, USA) unless otherwise stated. 


\section{Statistical Analysis}

Data are expressed as mean \pm standard deviation and were analyzed using the t-test, 1-way analysis of variance (ANOVA), and

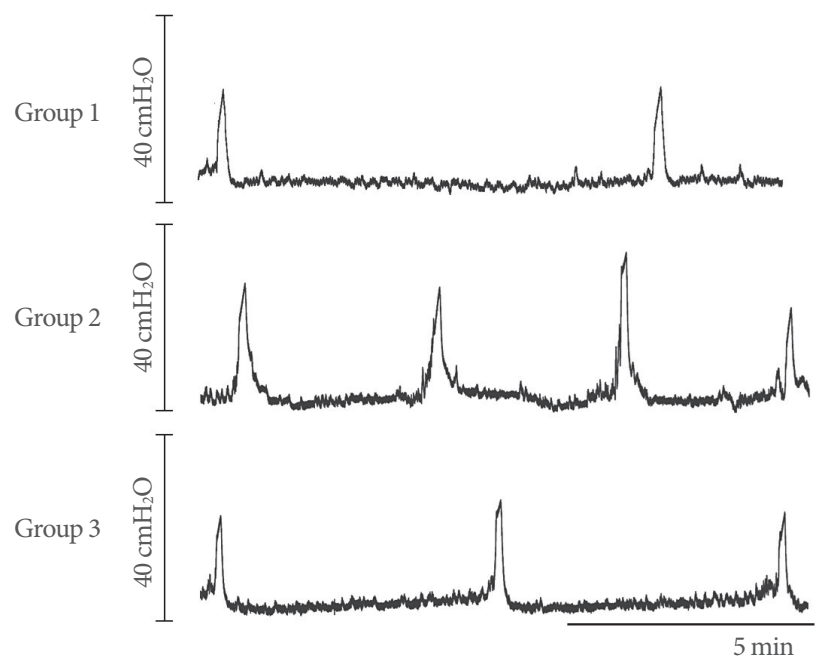

the Tukey multiple comparison test. IBM SPSS Statistics ver. 23.0 (IBM Co., Armonk, NY, USA) was used for statistical analyses. All statistical tests were 2-tailed, and P-values $<0.05$ were considered to indicate statistical significance.

\section{RESULTS}

\section{Effect of Neonatal Bladder Sensitization on Adult Bladder Function}

We found evidence of significant bladder overactivity in group 2 (Fig. 1). Specifically, the intercontraction interval was significantly lower in group $2(4.43 \pm 1.23$ minutes) than in groups 1 and 3 $(9.17 \pm 1.78$ and $6.58 \pm 1.98$ minutes, respectively). Bladder compliance was slightly higher in group $2\left(0.10 \pm 0.03 \mathrm{~mL} / \mathrm{cm} \mathrm{H}_{2} \mathrm{O}\right)$ than in groups 1 and $3\left(0.09 \pm 0.01\right.$ and $0.08 \pm 0.02 \mathrm{~mL} / \mathrm{cm} \mathrm{H}_{2} \mathrm{O}$, respectively. Moreover, maximum voiding pressure was also slightly higher in group $2\left(25.69 \pm 2.28 \mathrm{~cm} \mathrm{H}_{2} \mathrm{O}\right)$ than in groups 1 and $3\left(23.22 \pm 4.78\right.$ and $20.54 \pm 3.70 \mathrm{~cm} \mathrm{H}_{2} \mathrm{O}$, respectively).

\section{Evaluation of Adult Bladder Inflammation After Neonatal Bladder Sensitization}

To rule out the possibility that the sensitizing effects of acetic

Fig. 1. Representative cystometry results in adult rats. Group 1, normal control rats injected with saline; group 2, rats with chemically-induced cystitis; group 3, capsaicin-pretreated rats with chemically-induced cystitis.
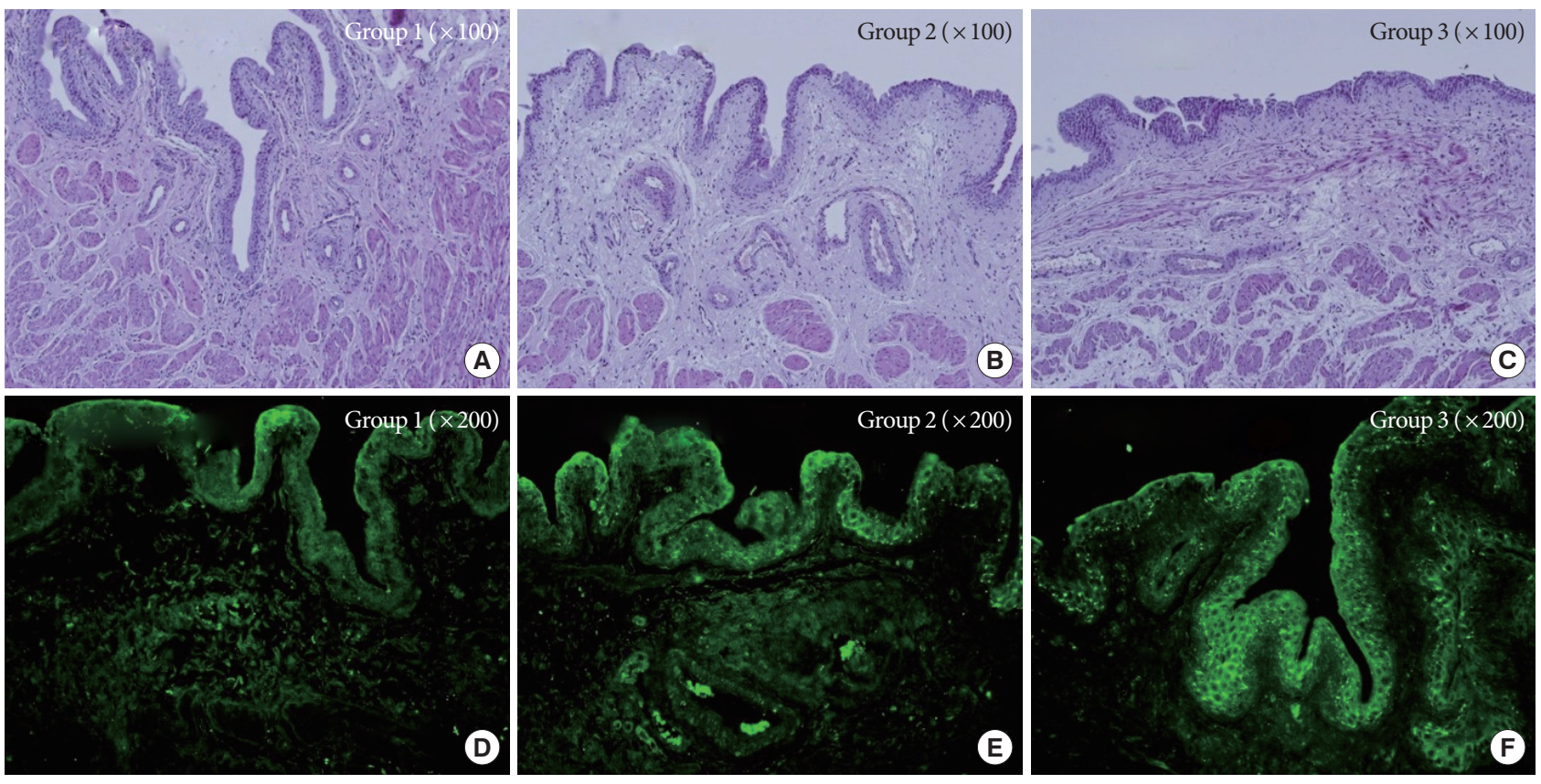

Fig. 2. Effects of neonatal acetic acid treatment of the bladder. (A-C) Photomicrographs of hematoxylin and eosin-stained sections of bladder tissue from adult rats. (D-F) Images of TRPV1 immunofluorescence (green) in sections of bladder tissue from adult rats. Group 1, normal control rats injected with saline; group 2, rats with chemically-induced cystitis; group 3, capsaicin-pretreated rats with chemically-induced cystitis. 
acid exposure were due to bladder inflammation, the bladders of adult rats were examined. Hematoxylin and eosin staining of bladder tissue showed no evidence of significant inflammation
(Fig. 2A-C). The IL-6 levels in adult groups 1 through 3 were $9.54 \pm 1.44,7.23 \pm 2.31$, and $6.24 \pm 1.99 \mathrm{pg} / \mathrm{mL}$, respectively. The IFN- $\gamma$ levels in adult groups 1 through 3 were $7.37 \pm 1.14$,
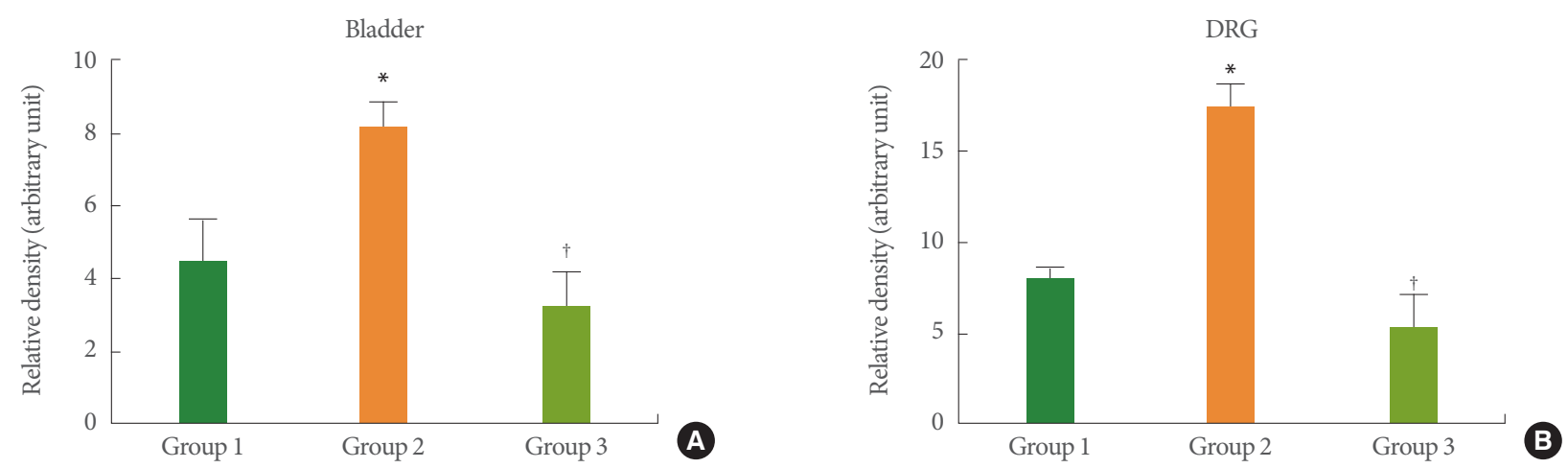

Fig. 3. Expression of TRPV1 mRNA in bladder tissue (A) and dorsal root ganglia (DRG) (B). P-values were obtained using the Tukey multiple comparison test. Group 1, normal control rats injected with saline; group 2, rats with chemically-induced cystitis; group 3 , capsaicin-pretreated rats with chemically-induced cystitis. ${ }^{*} \mathrm{P}<0.05$, statistical significance between the groups 1 and $2 .{ }^{\dagger} \mathrm{P}<0.05$, statistical significance between the groups 2 and 3.
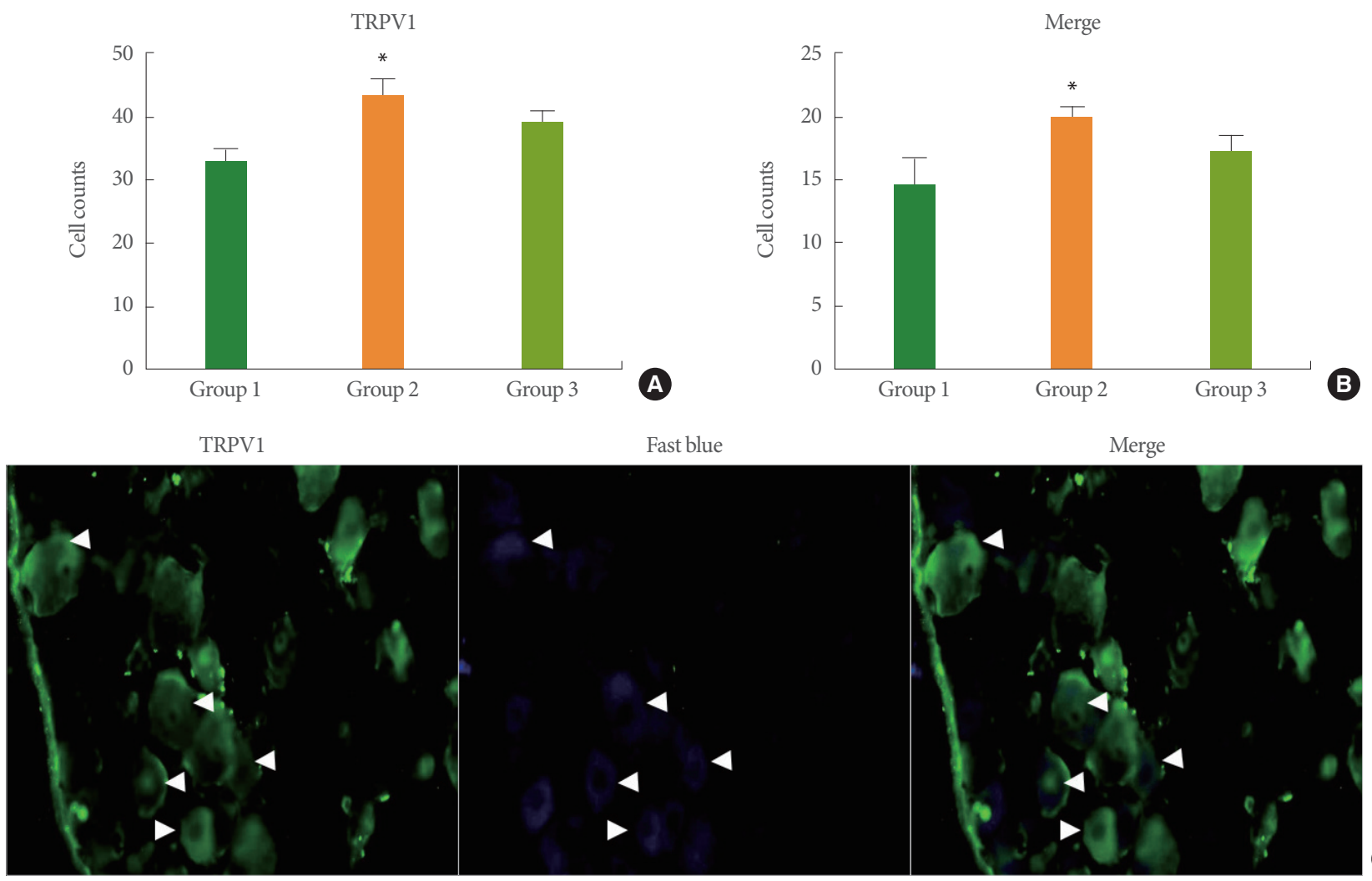

Fig. 4. TRPV1 expression in dorsal root ganglia (DRG) from adult rats. (A) Number of TRPV1-positive neurons in DRG. (B) Number of TRPV1-positive, Fast Blue (FB)-labeled neurons in DRG. (C) Images of TRPV1 immunofluorescence (green) in FB-labeled neurons (blue) in DRG. Triangles indicate TRPV1 immunofluorescence in FB-labeled neurons. Group 1, normal control rats injected with saline; group 2, rats with chemically-induced cystitis; group 3, capsaicin-pretreated rats with chemically-induced cystitis. P-values were obtained using the Tukey multiple comparison test. ${ }^{*} \mathrm{P}<0.05$, statistical significance between the groups 1 and 2 . 
$7.61 \pm 2.76$, and $8.56 \pm 2.22 \mathrm{pg} / \mathrm{mL}$, respectively. There were no significant differences in IL- 6 and IFN- $\gamma$ levels during adulthood.

\section{Adult TRPV1 Expression After Neonatal Bladder Sensitization}

To determine whether neonatal acetic acid treatment altered TRPV1 expression in adult rats, we measured TRPV1 expression in bladder and DRG tissue. Fig. 2D-F shows representative examples of TRPV1 expression in the bladder epithelium. Densitometric analysis relative to TRPV1 showed that TRPV1 expression was higher in groups 2 and 3 . We found that group 2 showed significantly higher expression of TRPV1 mRNA in bladder tissue and DRG than groups 1 and 3 (ANOVA, $\mathrm{P}<$ 0.05) (Fig. 3). The number of DRG neurons, identified by uptake of the retrograde label FB, that showed TRPV1 immunoreactivity was also significantly higher in group 2 than in group 1 , but there was no significant difference between group 2 and 3 (ANOVA, $\mathrm{P}<0.05$ ) (Fig. 4).

\section{DISCUSSION}

We demonstrated that bladder sensitization with dilute acetic acid during the neonatal period had long-lasting effects that continued until adulthood. Neonatal bladder sensitization decreased the intercontraction interval and increased TRPV1 expression in the adult bladder and DRG in the absence of histologic evidence of inflammation. This persistent bladder overactivity was attenuated by neonatal desensitization with systemic capsaicin treatment. These results suggest that the TRPV1 channel plays a role in generating and maintaining long-lasting bladder overactivity, even in the absence of inflammation.

Many studies have reported changes in cystometry and TRPV1 expression in acetic acid-induced cystitis models utilizing adult rats. Our cystometric results are consistent with those of Kitagawa et al. [11], who reported cystometric changes after the intravesical infusion of acetic acid in 10-week-old rats. They reported that the intravesical infusion of acetic acid decreased the intercontraction interval but did not obviously affect the maximal voiding pressure, and this decreased intercontraction interval was reversed by the TRPV1 antagonist JTS-653 [11]. These findings suggest that cystitis induced by acetic acid is associated with increased TRPV1 activity in neurons innervating the bladder.

Our model differs from previous rat models of acetic acidinduced cystitis [12-14]. In our model, dilute acetic acid was in- jected into the bladder of 10-day-old rat pups. The goal was to investigate the role of TRPV1 not only in the initiation of neonatal sensitization, but also in its maintenance. Therefore, we performed cystometry and evaluated TRPV1 expression in both the bladder and DRG of 8-week-old rats. When cystitis is induced by chemical irritants in rats, it is unknown whether bladder sensitization is due to inflammation or is a direct effect of acetic acid itself. Although TRPV1-deficient mice do not develop bladder overactivity in response to chemical irritants, histological examination of the bladder has shown similar inflammatory changes in both TRPV1-deficent and wild-type mice [15]. This implies that TRPV1 is associated with bladder overactivity, not cystitis. TRPV1 activation after dilute acetic acid administration might be related to the direct effect of acetic acid on sensory nerves or the local release of pro-algesic substances such as nerve growth factor [16], neuropeptides [17], anandamide [17], or adenosine triphosphate [18]. Our results suggest that TRPV1 is involved in both the initiation and maintenance of bladder sensitization.

TRPV1 is mostly confined to capsaicin-sensitive C fibers, which are unmyelinated, small-diameter primary afferent fibers. The bladder is rich with vanilloid-sensitive afferent fibers that detect bladder distension or the presence of chemical irritants [19]. We investigated whether blockade of capsaicin-sensitive C fibers during the neonatal period could prevent the initiation and maintenance of bladder sensitization induced by acetic acid. Capsaicin, a strong TRPV1 agonist, potently activates and desensitizes bladder afferent neurons by depleting neuropeptide stores in nerve endings [20]. In this manner, the afferent limb of the spinal reflux would be impaired. We desensitized capsaicinsensitive $\mathrm{C}$ fibers using systematic capsaicin treatment. In our model, large systemic doses (100-mg/kg body weight) were administered. Large doses of capsaicin produce long-term changes in the expression and function of TRPV1 in rats [21]. We found that neonatal desensitization with capsaicin treatment significantly decreased the intercontraction interval 8 weeks after bladder sensitization with acetic acid. This indicates that neonatal desensitization could reverse the bladder overactivity induced by acetic acid. Neonatal desensitization also significantly decreased the expression of TRPV1 mRNA in both the bladder and DRG, indicating that neonatal desensitization could antagonize TRPV1 activation. The molecular basis of the maintenance of bladder overactivity is not known. However, our results provide the first insight into the role of capsaicin-sensitive $\mathrm{C}$ fibers in the initiation and maintenance of bladder overactivity. 
A previous study showed that overactivity of the adult bladder was not maintained for 4 weeks after bladder sensitization with acetic acid [22]. In our model, bladder overactivity was maintained for 8 weeks without additional stimulation with acetic acid. This suggests that the nervous system may be vulnerable to bladder stimulation in the neonatal period, perhaps because the relevant regulatory systems have not fully matured. There is clinical evidence that childhood urinary symptoms and urinary tract infections are associated with adult overactive bladder [1]. It is possible that the persistence of this vulnerability may predispose individuals to bladder overactivity, even in adulthood. However, only a small percentage of children develop bladder overactivity as adults. Further experiments could help identify additional factors that contribute to neonatal vulnerability.

After a report on the role of the TRPV1 receptor in urinary tract pathology, several studies have described the effects of various TRPV1 antagonists [23-25]. One study showed lower amplitudes of bladder contraction after treatment with the TRPV1 antagonist GRC-6211 in rats with bladder overactivity [25]. Another study showed that pretreatment with the selective TRPV1 antagonist SB-366791 extinguished bladder overactivity in rats with cyclophosphamide-induced cystitis. These treatments focused on bladder sensitization in the neonatal period and its maintenance in adult period, not only by blocking its development, but also by discontinuing its maintenance. Our study showed that capsaicin-sensitive $\mathrm{C}$ fibers were involved in the maintenance of bladder sensitization. It is still unknown whether the previously studied TRPV1 antagonists are effective in blocking the maintenance of bladder sensitization. However, we found that bladder overactivity in adulthood was associated with the initiation and maintenance of TRPV1 expression and that the proper modulation of afferent activity of $\mathrm{C}$ fibers prevented bladder overactivity. Further studies are needed to investigate treatment methods that block afferent activity of $\mathrm{C}$ fibers.

In conclusion, we found a significant association between neonatal bladder sensitization and bladder overactivity in adult rats. This bladder overactivity in adult rats was associated with increased TRPV1 expression. Bladder overactivity was prevented by the blockade of capsaicin-sensitive $\mathrm{C}$ fibers during the neonatal period. Although we did not demonstrate the molecular basis of the initiation and maintenance of TRPV1 activity, our findings suggest that TRPV1 activation and its maintenance in the neonatal period could be a cause of overactive bladder symptoms in adults.

\section{AUTHOR CONTRIBUTION STATEMENT}

- Full access to all the data in the study and takes responsibility for the integrity of the data and the accuracy of the data analysis: JSP, $M H J, C H H$

- Study concept and design: JSP, $M H J, C H H$

- Acquisition of data: $\mathrm{MHJ}, \mathrm{HDJ}, \mathrm{YSC}, \mathrm{CHH}$

- Analysis and interpretation of data: JSP, $M H J, C H H$

- Drafting of the manuscript: JSP, $C H H$

- Critical revision of the manuscript for important intellectual content: $H D J$, YSC, $\mathrm{CHH}$

- Statistical analysis: JSP, $M H J$

- Obtained funding: $\mathrm{CHH}$

- Administrative, technical, or material support: $\mathrm{CHH}$

- Study supervision: $\mathrm{CHH}$

\section{ACKNOWLEDGEMENTS}

The authors would like to thank Dong-Su Jang, MFA, (Medical Illustrator) for his help with the illustrations.

\section{REFERENCES}

1. Fitzgerald MP, Thom DH, Wassel-Fyr C, Subak L, Brubaker L, Van Den Eeden SK, et al. Childhood urinary symptoms predict adult overactive bladder symptoms. J Urol 2006;175(3 Pt 1):989-93.

2. Held PJ, Hanno PM, Wein AJ, Pauly MV, Cahn MA. Epidemiology of interstitial cystitis: 2. In: Hanno PM, Staskin DR, Krane RJ, Wein AJ, editors. Interstitial cystitis. London: Springer; 1990. p. 29-48.

3. Hannestad YS, Lie RT, Rortveit G, Hunskaar S. Familial risk of urinary incontinence in women: population based cross sectional study. BMJ 2004;329:889-91.

4. Rohr G, Kragstrup J, Gaist D, Christensen K. Genetic and environmental influences on urinary incontinence: a Danish populationbased twin study of middle-aged and elderly women. Acta Obstet Gynecol Scand 2004;83:978-82.

5. Birder LA, Kanai AJ, de Groat WC, Kiss S, Nealen ML, Burke NE, et al. Vanilloid receptor expression suggests a sensory role for urinary bladder epithelial cells. Proc Natl Acad Sci U S A 2001;98:13396-401.

6. Avelino A, Cruz C, Nagy I, Cruz F. Vanilloid receptor 1 expression in the rat urinary tract. Neuroscience 2002;109:787-98.

7. Brady CM, Apostolidis AN, Harper M, Yiangou Y, Beckett A, Jacques TS, et al. Parallel changes in bladder suburothelial vanilloid receptor TRPV1 and pan-neuronal marker PGP9.5 immunoreactivity in patients with neurogenic detrusor overactivity after intra- 
vesical resiniferatoxin treatment. BJU Int 2004;93:770-6.

8. Apostolidis A, Brady CM, Yiangou Y, Davis J, Fowler CJ, Anand P. Capsaicin receptor TRPV1 in urothelium of neurogenic human bladders and effect of intravesical resiniferatoxin. Urology 2005;65:400-5.

9. Abrams P, Cardozo L, Fall M, Griffiths D, Rosier P, Ulmsten U, et al. The standardisation of terminology of lower urinary tract function: report from the Standardisation Sub-committee of the International Continence Society. Am J Obstet Gynecol 2002;187:11626.

10. Dinis P, Charrua A, Avelino A, Yaqoob M, Bevan S, Nagy I, et al. Anandamide-evoked activation of vanilloid receptor 1 contributes to the development of bladder hyperreflexia and nociceptive transmission to spinal dorsal horn neurons in cystitis. J Neurosci 2004; 24:11253-63.

11. Kitagawa Y, Wada M, Kanehisa T, Miyai A, Usui K, Maekawa M, et al. JTS-653 blocks afferent nerve firing and attenuates bladder overactivity without affecting normal voiding function. J Urol 2013; 189:1137-46.

12. Ishihama H, Momota Y, Yanase H, Wang X, de Groat WC, Kawatani $\mathrm{M}$. Activation of alpha1D adrenergic receptors in the rat urothelium facilitates the micturition reflex. J Urol 2006;175:358-64.

13. Mitsui T, Kakizaki H, Matsuura S, Tanaka H, Yoshioka M, Koyanagi $\mathrm{T}$. Chemical bladder irritation provokes c-fos expression in the midbrain periaqueductal gray matter of the rat. Brain Res 2003; 967:81-8.

14. Fraser MO, Chuang YC, Tyagi P, Yokoyama T, Yoshimura N, Huang L, et al. Intravesical liposome administration--a novel treatment for hyperactive bladder in the rat. Urology 2003;61:656-63.

15. Dornelles FN, Andrade EL, Campos MM, Calixto JB. Role of CXCR2 and TRPV1 in functional, inflammatory and behavioural changes in the rat model of cyclophosphamide-induced haemorrhagic cystitis. Br J Pharmacol 2014;171:452-67.

16. Bonnington JK, McNaughton PA. Signalling pathways involved in the sensitisation of mouse nociceptive neurones by nerve growth fac- tor. J Physiol 2003;551(Pt 2):433-46.

17. Harrison S, De Petrocellis L, Trevisani M, Benvenuti F, Bifulco M, Geppetti P, et al. Capsaicin-like effects of N-arachidonoyl-dopamine in the isolated guinea pig bronchi and urinary bladder. Eur J Pharmacol 2003;475:107-14.

18. Pandita RK, Andersson KE. Intravesical adenosine triphosphate stimulates the micturition reflex in awake, freely moving rats. J Urol 2002;168:1230-4.

19. Du S, Araki I, Yoshiyama M, Nomura T, Takeda M. Transient receptor potential channel $\mathrm{Al}$ involved in sensory transduction of rat urinary bladder through C-fiber pathway. Urology 2007;70:826-31.

20. Dussor GO, Helesic G, Hargreaves KM, Flores CM. Cholinergic modulation of nociceptive responses in vivo and neuropeptide release in vitro at the level of the primary sensory neuron. Pain 2004; 107:22-32.

21. Szallasi A, Blumberg PM. Vanilloid (Capsaicin) receptors and mechanisms. Pharmacol Rev 1999;51:159-212.

22. Al-Chaer ED, Kawasaki M, Pasricha PJ. A new model of chronic visceral hypersensitivity in adult rats induced by colon irritation during postnatal development. Gastroenterology 2000;119:127685.

23. Santos-Silva A, Charrua A, Cruz CD, Gharat L, Avelino A, Cruz F. Rat detrusor overactivity induced by chronic spinalization can be abolished by a transient receptor potential vanilloid 1 (TRPV1) antagonist. Auton Neurosci 2012;166:35-8.

24. Cefalu JS, Guillon MA, Burbach LR, Zhu QM, Hu DQ, Ho MJ, et al. Selective pharmacological blockade of the TRPV1 receptor suppresses sensory reflexes of the rodent bladder. J Urol 2009;182:77685.

25. Charrua A, Cruz CD, Narayanan S, Gharat L, Gullapalli S, Cruz F, et al. GRC-6211, a new oral specific TRPV1 antagonist, decreases bladder overactivity and noxious bladder input in cystitis animal models. J Urol 2009;181:379-86. 